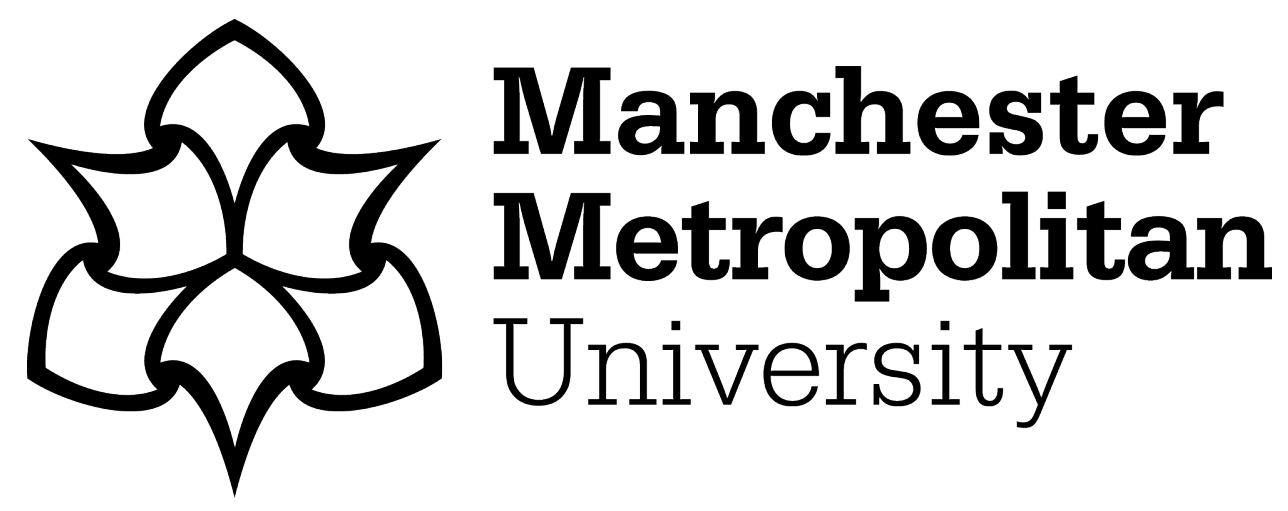

Malic, Sladjana, Rai, Sheela, Redfern, James, Pritchett, James, Liauw, Christopher, Verran, Joanna and Tosheva, Lubomira (2018) Zeoliteembedded silver extends antimicrobial activity of dental acrylics. Colloids and Surfaces B: Biointerfaces, 173. pp. 52-57. ISSN 0927-7765

Downloaded from: https://e-space.mmu.ac.uk/621494/

Version: Accepted Version

Publisher: Elsevier

DOI: https://doi.org/10.1016/j.colsurfb.2018.09.043

Usage rights: Creative Commons: Attribution-Noncommercial-No Derivative Works 4.0

Please cite the published version 


\title{
Zeolite-embedded silver extends antimicrobial activity of dental acrylics
}

Sladjana Malic,* Sheela Rai, James Redfern, James Pritchett, Christopher M. Liauw, Joanna Verran and Lubomira Tosheva* Faculty of Science and Engineering, Manchester Metropolitan University, Chester Street, Manchester, M1 5GD, United Kingdom. E-mail: 1.tosheva@mmu.ac.uk

Statistical summary: 5547 words; 1 table, 5 figures

\begin{abstract}
The insertion of prosthetic devices into the oral cavity affects the oral microflora and results in accumulation of microorganisms on the prosthetic surface. Such fouling of denture surfaces can lead to a number of oral diseases and consequently to the replacement of the denture. Here, we report the post-synthesis introduction of silver in zeolite-loaded dental acrylic (DAZ) resins that does not influence the mechanical or aesthetic properties of the DA resins, and provides them with a long-term antimicrobial activity. Na-FAU zeolite ( $2 \mathrm{wt} \%$ ) was incorporated into DA resin, which was conventionally processed and cut into $10 \mathrm{~mm}$ × $20 \mathrm{~mm}$ × $3 \mathrm{~mm}$ coupons. The $\mathrm{Na}^{+}$in the zeolite was then exchanged with $\mathrm{Ag}^{+}$via immersion of the DAZ coupons in $0.01 \mathrm{M}$ $\mathrm{AgNO}_{3}$ solution to obtain DAZ/Ag-treated coupons used in antimicrobial tests. Antimicrobial tests showed that the DAZ/Ag-treated coupons were active against Candida albicans (a reference and a clinically relevant strain), Streptococcus mutans and Fusobacterium nucleatum. Ag leaching tests on the Ag-charged coupons at 1, 2, 3, $4,7,14,30$ and 45 days of incubation in distilled water at $37^{\circ} \mathrm{C}$, indicated sustained
\end{abstract}


release of silver. Antimicrobial tests using a reference Candida albicans strain showed that the leached coupons retained antimicrobial activity after 45 days immersion in distilled water, but, after 60 days incubation no antimicrobial activity was observed. Cytotoxicity assay results indicated that the DAZ/Ag-treated coupons showed no additional cytotoxicity compared to neat dental acrylic coupons.

Keywords: Dental acrylic resins, Zeolites, Ag ion-exchange, Long-term antimicrobial dentures, Candida albicans 


\section{Introduction}

Oral diseases such as dental caries, periodontal diseases and oral candidosis cause significant health problems [1,2]. Management of oral candidosis is limited due to the low number of antifungal drugs available, their relatively high toxicity and the emergence of antifungal resistance $[3,4]$. Non-rigorous denture hygiene facilitates biofilm build up and initiates local inflammatory responses in the palatal mucosa $[5,6]$. The denture itself affects salivary flow and allows more niches for microorganisms to flourish, co-aggregate and form biofilms, leading to malodor and a potential reservoir of respiratory pathogens with an increased risk of pulmonary infections [7-10]. It has been estimated that by 2025, in the EU alone, $53 \%$ of the population will be over 65 years of age, resulting in significant challenges for oral healthcare delivery [11]. An increasing elderly population will lead to increased demand for dentures. Thus, it would be worthwhile to develop methods for fabrication of dentures that contain controlled release antimicrobials to address the above-mentioned problems.

Heat-cured dental acrylic resin is composed of powder and liquid components (typical ratio; ca. 2.5:1.0 by mass powder to liquid) [12]. The powder component consists of polymethylmethacylate (PMMA) suspension polymer powder, with accessible dibenzoyl peroxide (DBP) initiator; aesthetics related additives such as pigments, opacifiers and flock fibres are also added. Depending on the mode of manufacture, the DBP present is residual from the suspension polymerization and/or is added along with the other additives. The liquid component is composed of inhibited methylmethacrylate (MMA) monomer combined with ethyleneglycoldimethacrylate (EGDMA) crosslinking agent (ca. $8 \mathrm{wt} \%$ ). Due to some dissolution of the PMMA beads, a dough like mass is formed a short period after mixing the powder and liquid 
components. The dough is pressed into the mold and the liquid component polymerized by heating the filled mold, following a carefully planned temperature program. The molding surface is finished using a range of abrasives and then polished. Due to the presence of a significant proportion of incompletely dissolved PMMA suspension polymer particles in a matrix that consists of a mixture of uncrosslinked (dissolved from the PMMA powder particles) and EGDMA crosslinked PMMA chains (from the polymerized liquid component), a dental acrylic surface is heterogeneous in nature even before exposed pigment/opacifier particles and flock fibers are considered. The maximum water sorption permissible of dental acrylic according to BS EN ISO 207951:2013 is $32 \mu \mathrm{g} \mathrm{mm}^{-3}$ which translates to about $2.7 \mathrm{wt} \%$ according to Kusy et al. [13]. Cast acrylic sheet has water sorption values reported to be between $0.2 \mathrm{wt} \%$ and $2 \mathrm{wt} \%$ after very long exposure periods (Perspex design guide, Darwen, UK). The heterogeneous surface of dental acrylic combined with the relatively high water sorption therefore renders it more vulnerable to surface fouling by microbial species than cast acrylic sheet. These conditions enable the growth of Candida which is adept at adhering and forming a biofilm [5,14]. The Candida genus, and C. albicans in particular, plays a very important part in biofilm formation and denture stomatitis, however, bacteria also coexist within denture biofilms [9, 15-20]. Streptococcus mutans (S. mutans) and Fusobacterium nucleatum (F. nucleatum) have been found in denture biofilms [9], making them relevant to studies of antimicrobial dental materials.

Zeolites are microporous aluminosilicate materials with regular pore structures and high surface areas, which can slowly release pre-loaded antimicrobials over long periods of time [21]. Their ion-exchange properties can be used to introduce antimicrobial metals into zeolite-loaded dental acrylics. Silver has been selected in this 
study because of its broad spectrum of antimicrobial activity $[22,23]$. The aims of the present work were to develop a method for fabrication of Ag-containing dental acrylic resins with long-term antimicrobial activity and a potential for recharging of Ag upon Ag depletion.

\section{Materials and methods}

\subsection{Preparation of dental acrylics}

Dental acrylic (DA) was prepared by gently mixing $30 \mathrm{~mL}$ liquid component (Pegasus Plus Heat Cure Monomer, Davis Schottlander \& Davis Ltd, UK) and $69 \mathrm{~g}$ powder component (Pegasus Plus Denture Base, pink veined, Davis Schottlander \& Davis Ltd, UK) in a glass vacuum jar. The powder - liquid mixture was allowed to rest for 6-8 minutes in order to allow a fraction of the PMMA particles to dissolve in the MMA and so confer a dough-like consistency to the mixture. The mixture was removed from the jar and kneaded, and then packed into eight $10 \mathrm{~mm} \times 110 \mathrm{~mm} \times 3 \mathrm{~mm}$ molds. Each mold was screwed down tightly and placed in a hydraulic press, applying 150 bar of pressure. After quickly removing the excess acrylic, the mold was again placed in the hydraulic press and pressed at 200 bar pressure The molding was cured at $95^{\circ} \mathrm{C}$ for $9 \mathrm{~h}$ in a dry heat-curing unit (Milnes Bros, UK). After cooling to room temperature, the moldings were released from the mold and cut into $10 \mathrm{~mm}$ x $20 \mathrm{~mm}$ x $3 \mathrm{~mm}$ coupons for antimicrobial tests.

Na-FAU zeolite was prepared from a gel with the molar composition $8 \mathrm{NaOH}$ : $0.2 \mathrm{Al}_{2} \mathrm{O}_{3}: 1.0 \mathrm{SiO}_{2}: 200 \mathrm{H}_{2} \mathrm{O}$ following the procedure described in ref. [24]. The zeolite was organically functionalized with 3-(trimethoxysilyl) propylmethacrylate (Alfa Aesar) prior to adding to the DA precursor mixture [25]. Na-FAU zeolite was mixed 
with $10 \mathrm{wt} \%$ silane solution in cyclohexane at a zeolite to solution weight ratio of 1 to 10, stirred for $30 \mathrm{~min}$ at room temperature, washed several times with cyclohexane and dried at $50{ }^{\circ} \mathrm{C}$ for $72 \mathrm{~h}$. Experiments were also performed using Ag-FAU zeolite prepared by ion-exchange with $\mathrm{AgNO}_{3}$ solution $\left(0.05 \mathrm{M} \mathrm{AgNO}_{3}\right.$ solution added at a zeolite to solution weight ratio of 1 to 20 , stirred for $72 \mathrm{~h}$ in the dark, rinsed repeatedly with distilled water and dried at $60{ }^{\circ} \mathrm{C}$ overnight), followed by organic functionalization [25]. Silane-treated Ag-FAU zeolite was added to uncured DA at 0.2, 0.4, 0.7 and 2 wt $\%$, whereas Na-FAU and silane-treated Na-FAU zeolite was added to uncured DA at $2 \mathrm{wt} \%$ for post-synthesis silver introduction.

Na-FAU-loaded (silane-modified Na-FAU) DA coupons were treated with $\mathrm{AgNO}_{3}$ solution to ion-exchange $\mathrm{Na}$ with $\mathrm{Ag}$ to obtain DAZ/Ag-treated coupons. Individual dental acrylic coupons were transferred into $25 \mathrm{~mL}$ universal bottles and 20 $\mathrm{mL}$ of $0.01 \mathrm{M} \mathrm{AgNO}_{3}\left(\mathrm{AgNO}_{3}\right.$, Alfa Aesar) solution was added to each universal. The bottles were covered with aluminum foil, and the samples were incubated on a shaker at $150 \mathrm{rpm}$ at room temperature for $24 \mathrm{~h}$. After incubation, the samples were washed 20 times with distilled water by decanting and were dried overnight at $60{ }^{\circ} \mathrm{C}$. Blank DA coupons were treated in the same way to distinguish between silver ion-exchange in the zeolite present in the composite and silver absorption by the DA.

\subsection{Characterization of dental acrylics}

Zeolite $10 \mathrm{~mm} \times 50 \mathrm{~mm} \times 2 \mathrm{~mm}$ test pieces were band sawn from the dental acrylic sheets that were prepared as previously described. The long edges of the test pieces were smoothed using water-cooled metallographic grinding decks fitted with progressively finer silicon carbide paper (240-400-600 grit). Thickness and widths of test pieces were measured using a digital caliper. Flexural properties (modulus and 
strength) of DA and DA loaded with $2 \mathrm{wt} \% \mathrm{Na}-\mathrm{FAU}$ or $2 \mathrm{wt} \% 3$-(trimethoxysilyl) propylmethacrylate-treated Na-FAU and Ag-FAU were determined using a Hounsfield H10KS; span was $32 \mathrm{~mm}$ and cross-head speed was $4 \mathrm{~mm} \mathrm{~min}^{-1}$. Flexural modulus was determined via linear regression of the load deflection data between limits of 0.3 and 0.8 $\%$, respectively. Flexural strength was the maximum flexural stress recorded. Six test pieces of each material were tested and the averages and standard deviations recorded. Statistical analysis was performed with Prism version 7 (GraphPad Software, La Jolla, CA, USA). One-way ANOVA followed by Tueky's multiple comparisons test was used to evaluate statistical differences between the samples at $95 \%$ confidence.

Scanning electron analysis of DA and DAZ/Ag-treated coupons was performed with a Carl Zeiss Ltd 40VPSupra Scanning Electron Microscope. Coupons were mounted on $\mathrm{Al}$ stubs and coated with gold prior analysis. For cross-section images, the coupons were attached perpendicularly to the Al stubs with a cement glue.

\subsection{Microorganisms}

In total, four microorganisms were used for the antimicrobial testing, namely $C$. albicans NCYC 1363, C. albicans 135BM2/94, a clinical strain isolated from a patient attending the dental hospital at Cardiff University (ref. [26]), S. mutans NCTC 1083 and F. nucleatum KS 515-14. The two C. albicans strains were grown on Sabouraud Dextrose Agar (SDA; Oxoid, UK) and then sub-cultured in Sabouraud Dextrose Broth (SAB; Oxoid, UK) for $18 \mathrm{~h}$ at $37^{\circ} \mathrm{C}$ under aerobic conditions. S. mutans NCTC 10832 and F. nucleatum KS 515-14 were grown on Fastidious Anaerobe agar (FAA; Lab M Limited, UK) containing 5-10\% defibrinated horse blood (TCS Biosciences Ltd, UK) and then sub-cultured in Fastidious Anaerobe broth (FAB; Lab M Limited, UK) at 37 ${ }^{\circ} \mathrm{C}$ under anaerobic conditions. 


\subsection{Antimicrobial testing}

Antimicrobial tests were performed as described in our previous work [25]. Single colonies of C. albicans NCYC 1363 and C. albicans 135BM2/94 were subcultured from SDA in sterile SAB. The broth was incubated for $18 \mathrm{~h}$ at $37{ }^{\circ} \mathrm{C}$ on an orbital shaker (New Brunswick Scientific, UK; I-26 model) at $150 \mathrm{rpm}$. The liquid culture was centrifuged at $3000 \mathrm{rpm}$ (1721g; Sigma 3-16L model, UK) for $10 \mathrm{~min}$. Afterwards, the supernatant was discarded and the pellet re-suspended in sterile $1 / 4$ Ringer's solution (Oxoid, UK). An optical density of 1.0 at $540 \mathrm{~nm}$ was obtained (Jenway 6305 Spectrophotometer, UK), equaling approximately $10^{7} \mathrm{CFU} \mathrm{mL}^{-1}$. A 1:100 dilution was performed in Ringer's solution and the suspension (approximately. $10^{5}$ CFU mL $\mathrm{mL}^{-1}$ ) was used to inoculate the dental acrylic surfaces, which were laid flat in individual Petri dishes. $50 \mu \mathrm{L}$ of the standardized suspension (C. albicans NCYC 1363, $5 \times 10^{5} \mathrm{CFU} \mathrm{mL}^{-1}$; C. albicans $135 \mathrm{BM} 2 / 94,10 \times 10^{5} \mathrm{CFU} \mathrm{mL}^{-1} ;$ F. nucleatum KS 515$14,5 \times 10^{5} \mathrm{CFU} \mathrm{mL} \mathrm{m}^{-1}$; and $S$. mutans NCTC $10832,3 \times 10^{6} \mathrm{CFU} \mathrm{mL}^{-1}$ ) was pipetted onto each coupon's surface and a $20 \mathrm{~mm}$ x $10 \mathrm{~mm}$ polyethylene film was gently pressed on top of the droplet to ensure the cell suspension were spread evenly on the surface. Immediately after inoculation, three coupons were placed individually into $10 \mathrm{~mL}$ neutralizing agent $(14.6 \%$ sodium thiosulphate and $10 \%$ sodium thioglycolate in 100 $\mathrm{mL}$ distilled water; $5 \mathrm{~mL}$ was filter sterilized into $495 \mathrm{~mL}$ sterile distilled water) and vortexed for $30 \mathrm{~s}$. Serial dilutions were prepared and $100 \mu \mathrm{L}$ aliquots were spread onto SAB agar plates, incubated at $37^{\circ} \mathrm{C}$ overnight and the $\mathrm{CFU}$ counted. The remaining coupons in petri dishes were incubated at $37^{\circ} \mathrm{C}$ and tested as above after 1 and 5 h. $S$. mutans NCTC 10832 and F. nucleatum KS 515-14 were cultured under anaerobic conditions. The agar plates for the CFU counting of F. nucleatum KS 515-14 were 
incubated for $48 \mathrm{~h}$. The tests were carried out in triplicate and experiments were repeated three times. The coupons tested were DA, DA treated with $\mathrm{AgNO}_{3}$ solution (DA/Ag-treated) and Na-FAU (silane-modified)-loaded (2 wt $\%$ ) DA after treatment with $\mathrm{AgNO}_{3}$ solution (DAZ/Ag-treated).

\subsection{Cytotoxicity assay method}

Immortalized human hepatic stellate cells (Lx-2 cells developed by Scott Friedman, Mount Sinai, NYC, ref. [27]) were cultured in monolayer at $5 \% \mathrm{CO}_{2}$ and 37 ${ }^{\circ} \mathrm{C}$ in Dulbecco's modified Eagle's medium plus L-glutamine, Na-pyruvate, and antibiotics supplemented with $10 \%$ fetal bovine serum. For experiments, Lx-2 cells were plated in 6 well plates in serum-free conditions, in the presence of DA or DAZ/Ag-treated coupons for $48 \mathrm{~h}$. Media was then recovered and incubated for $1 \mathrm{~h}$ at $37{ }^{\circ} \mathrm{C}$ with MTT (EMD Millipore, Billerica, MA), a colorimetric assay used to assess cytotoxicity of potentially toxic materials [28]. Purple formazan crystals, formed by active mitochondrial enzymes, were dissolved in $0.04 \mathrm{~N} \mathrm{HCl}$ in isopropanol (Fisher) and the intensity measured at $560 \mathrm{~nm}(\mathrm{n}=3$ experiments), with sterile growth media used as a blank measure.

\subsection{Ag release experiments}

Initial Ag leaching experiments were performed with DA/Ag-treated and DAZ/Ag-treated coupons, to distinguish between Ag, which remains adsorbed on / absorbed into dental acrylics after sample preparation, and Ag, which is ion-exchanged within the zeolite. Nine coupons of each type of sample were placed into universal bottles containing $20 \mathrm{~mL}$ sterile distilled water, and incubated at $37^{\circ} \mathrm{C}$ on a rotary shaker at $150 \mathrm{rpm}$. Coupons were transferred to fresh bottles with $20 \mathrm{~mL}$ sterile distilled water after 1, 2, 3, 4 and 7 days. All universal bottles were stored at $-20{ }^{\circ} \mathrm{C}$ prior to 
measurement of the Ag concentration using a Thermo Scientific iCAP6300 Duo inductively coupled plasma optical emission spectrometer (ICP-OES, Thermo Scientific, UK). Long-term silver release experiments using DAZ/Ag-treated coupons (in triplicate) were performed similarly; samples were taken after 1, 2, 3, 4, 7, 14, 30 and 45 days. To detect possible microbial contamination, a loop of sample was inoculated every $48 \mathrm{~h}$ onto sterile Tryptone Soya agar plates (Oxoid, UK) and incubated at $37^{\circ} \mathrm{C}$ for $18-24 \mathrm{~h}$. The coupons were tested against C.albicans NCYC 1363 as described above after 45 and $60 \mathrm{~d}$ of the Ag leaching experiments.

\section{Results and discussion}

Initially, DA was loaded with silane-treated Ag-FAU. However, these samples showed a brown coloration, which became more intense with increased loading of AgFAU (Fig. 1a). For dental applications, such a color change is unacceptable. This formation of dark coloration indicates that the $\mathrm{Ag}^{+}$had undergone reduction to elemental silver during free radical addition polymerization of the MMA. Similar results were observed in our previous work for Ag-FAU-loaded silicone elastomers [25]. Visual inspection indicated that post-fabrication treatment with silver nitrate of DA and Na-FAU-loaded DA coupons did not result in such color change and that the "natural" color of the DA coupons was thus retained (Fig. 1b). In addition, SEM analysis showed that the loading of zeolite-embedded silver did not significantly change the DA surface morphology (Supporting information, Fig. S1)

The characteristics of the Na-FAU and Ag-FAU zeolite used in this work have been discussed in detail in our previous publication [25]. The zeolite functionalization with silane was found to result in reduced zeolite aggregation and improved filler- 
matrix adhesion. Flexural modulus and flexural strength obtained for DA (control DA), DA loaded with Na-FAU (zeolite DA), DA loaded with silane-treated Na-FAU (Zeolite-silane DA) and DA loaded with silane-treated Ag-FAU (Ag zeolite-silane DA) are presented in Table 1. Addition of the unmodified zeolite (Na-FAU) and silanetreated Na-FAU had no statistically significant effect on flexural modulus, relative to the control DA. However, silane treatment of Na-FAU lead to a statistically significant $(\mathrm{P}=0.003)$ increase in flexural modulus relative to untreated $\mathrm{Na}-\mathrm{FAU}$, thus supporting an increase in filler - matrix adhesion. Interestingly, addition of silane-treated Ag-FAU to DA led to a significant increase in flexural modulus, relative to the control DA ( $\mathrm{P}<$ $0.0001)$ and to silane-treated $\mathrm{Na}-\mathrm{FAU}(\mathrm{P}=0.02)$. The latter may indicate that Ag-FAU may interact more prolifically with the silane than Na-FAU, leading to further increased filler - matrix adhesion. Addition of all types of zeolite led to reductions in flexural strength relative to the control DA. This was most likely due to incomplete dispersion of the zeolites. In all cases, however, the measured properties satisfied BS EN ISO 207951:2013, which specifies that flexural modulus of denture base acrylic must not be lower than $2 \mathrm{GPa}$ and flexural strength not lower than $65 \mathrm{MPa}$.

Initial antimicrobial tests using the reference $C$. albicans strain showed that the DA/Ag-treated and DAZ/Ag-treated samples had similar antimicrobial activity (Supporting information, Fig. S2). This indicated that silver was released from both the zeolite-free and the zeolite-loaded dental acrylics, resulting in an antimicrobial activity. Short-term silver leaching experiments were performed to determine a procedure for the removal of the 'non-zeolitic' silver from DA prior to testing. The results in Supporting information, Fig. S3, showed that the adsorbed silver was removed after $2 \mathrm{~d}$ of incubation in distilled water. The antimicrobial test using a $2 \mathrm{~d}$-leached sample 
(Supporting information, Fig. S4) confirmed this conclusion and the DA/Ag-treated in this case did not kill C. albicans after 1 and $5 \mathrm{~h}$ of incubation. Further antimicrobial tests were performed after $2 \mathrm{~d}$ immersion of the DA/Ag-treated and DAZ/Ag-treated coupons in distilled water.

The antimicrobial activity of the post molding Ag-charged Na-FAU-loaded DA (DAZ/Ag-treated) coupons against the two strains of C. albicans as well as $S$. mutans and F. nucleatum are shown in Fig. 2. DAZ/Ag-treated coupons showed antimicrobial activity against C. albicans NCYC 1363 (Fig. 2a, 3-log reduction in microbial counts after 5 h of incubation), C. albicans 135BM2/94 (Fig. 2b, 3-log reduction in microbial counts after 5 h of incubation), S. mutans (Fig. 2c, 4-log reduction in bacterial counts after $5 \mathrm{~h}$ of incubation) and $F$. nucleatum (Fig. 2d, 3-log reduction in bacterial counts after $5 \mathrm{~h}$ of incubation). In all cases, the DA and DA/Ag-treated coupons did not show activity against the selected microorganisms in the tests.

Cell viability tests were performed to assess the biocompatibility of the DAZ/Ag-treated samples. Absorbance measured using the MTT assay is an indicator of mitochondrial enzyme activity. There was no significant difference between the DA and DAZ/Ag-treated (Fig. 3). Therefore, compared to DA, DAZ/Ag-treated does not demonstrate any additional cytotoxicity.

Long-term silver leaching experiments were performed to determine the antimicrobial lifetime of the DAZ/Ag-treated coupons. The results shown in Fig. 4 indicate that there was a sustained Ag release over the time period studied (45 d), although the concentration of released $\mathrm{Ag}$ gradually decreased from $1.89 \mathrm{ppm}$ (after $1 \mathrm{~d}$ of incubation) to $0.12 \mathrm{ppm}$ (after $45 \mathrm{~d}$ of incubation). Owing to the significance of Candida in causing denture stomatitis [4], this microorganism was used to determine 
the long-term antimicrobial activity of DAZ/Ag-treated samples. The antimicrobial tests against $C$. albicans $135 \mathrm{BM} 2 / 94$ performed after $45 \mathrm{~d}$ of leaching showed that the antimicrobial activity of DAZ/Ag-treated coupons was retained (Fig. 5). However, the activity was lost after $60 \mathrm{~d}$ of leaching indicating that Ag recharging would be needed after ca. $45 \mathrm{~d}$ to restore the antimicrobial activity of DAZ/Ag-treated (Fig. 5).

The treatment of denture-related stomatitis involves a range of different approaches such as antifungal therapy (both systemic and topical application), use of disinfectants and cleansers, laser treatment of palatal tissue, ongoing oral hygiene protocols for patients, denture replacement, placement of a resilient liner or a tissue conditioner and microwave disinfection [29]. This multi-modal approach aims to eliminate pathogenic microorganisms and prevent/disrupt biofilm formation. Figueiral et al. have shown in their pilot study that antifungal therapy in isolation did not eliminate denture stomatitis [30]. Treatment of patients with fluconazole resulted in some healing and reduced denture stomatitis symptoms, but the improvement was short lived; after a 6 month follow-up the severity of the condition had returned to pretreatment levels. Furthermore, patients had developed a certain level of antimicrobial resistance to fluconazole. A more elegant approach to prevent denture stomatitis is to incorporate antimicrobials into denture base materials [31], or to functionalize such materials in order to increase drug binding capacity [32]. Silver has been used for centuries for its antimicrobial effect in different fields of medicine [14,33]. Previous studies using PMMA-silver nanoparticle discs on NIH-3T3 mouse embryonic fibroblasts and a Jurkat human lymphocyte cell line have shown that metabolism and proliferation have not been affected [34] and that silver does not present a threat to human health [14]. However, drawbacks associated with the use of silver nanoparticles 
and other silver-based materials include nanoparticle aggregation and deterioration of composite mechanical properties, aesthetic incompatibility due to color changes resulting from reduction of $\mathrm{Ag}^{+}$to $\mathrm{Ag}^{0}$ and short-term antimicrobial activity $[35,36]$. Further critical considerations include long-term studies, which typically include reports of the antimicrobial activity after up to $28 \mathrm{~d}$ without precise determination of the antimicrobial lifetime of the dental materials $[37,38]$. The possibility to recharge the antimicrobial agent into the denture material has also rarely been demonstrated [39].

The present study addresses many of the above problems. A novel method for post-fabrication charging of silver was developed through utilization of the ionexchange capacity of a zeolite, which has been loaded into dental acrylic resins prior to curing. As this method eliminates the risk of $\mathrm{Ag}^{+}$reduction during acrylic polymerization, the tendency to develop coloration in dental acrylic resins was also eliminated to the extent that post-fabrication charging did not affect visually the color of the material. Charging of silver can be potentially repeated after silver depletion by immersion in silver nitrate solution, thus providing a restoration of antimicrobial activity and possibly significantly extended denture lifetime. The flexural strength and flexural modulus requirements for denture base acrylics of the zeolite-loaded DA were still met. Finally, the zeolite-loaded DA did not demonstrate additional cytotoxicity compared to neat DA.

In terms of antimicrobial properties, DAZ/Ag-treated samples showed consistent activity against a reference and a clinical strain of $C$. albicans as well as $S$. mutans and F. nucleatum. This activity can be attributed to the silver ions released from the DAZ/Ag-treated coupons. The silver release experiments revealed that during the first two days of leaching the silver came in to solution via two routes; the rapid reduction in 
silver concentration was due to depletion of silver that was adsorbed within the DA resin matrix coupled with the more sustained release of silver from the zeolite particles. The latter zeolite-related mode of silver delivery continued far beyond two days. The DAZ/Ag-treated coupons were soaked in sterile distilled water for $2 \mathrm{~d}$ prior to tests in order to ensure that the silver came only from the zeolite. However, for practical applications, this is not necessary and DAZ/Ag-treated samples can be used immediately after preparation. Further, the $45 \mathrm{~d}$ silver release experiments and the antimicrobial tests indicated that the antimicrobial lifetime of the DA materials with respect to C. albicans was about $45 \mathrm{~d}$, during which their antimicrobial potential remained constant, although activity was lost after $60 \mathrm{~d}$.

The results presented are significant in terms of the development of a procedure for modifying denture base materials with potentially rechargeable antimicrobial silver by simple $\mathrm{AgNO}_{3}$ treatment. Oral health is a problem affecting a large proportion of the ever-increasing elderly population [9]. It is important to highlight that there is a systematic link between oral health and other diseases such as cardiovascular diseases [40], kidney diseases [41], and pulmonary diseases [42], and therefore a management strategy on how to combat oral health, including denture stomatitis, is of utmost importance. This study shows that addition of Ag to PMMA inhibits the growth of potential pathogenic oral microorganisms (bacteria and fungi) and could be used for management of oral health in patients with dentures.

Future developments of this study would include testing of single species biofilm assays on DAZ/Ag-treated coupons, tests using physiological conditions such as artificial saliva, and tests against a consortium of oral microorganisms associated with denture plaque. Charging of silver can be potentially repeated after silver depletion, thus 
providing a restoration of antimicrobial activity and possibly significantly extended denture lifetime. Determination of the number of cycles this can be done without affecting performance would be worth studying.

\section{Conclusion}

A method was developed to prepare silver-containing dental acrylic resins with antimicrobial activity against fungi (clinical and reference strains of $C$. albicans) and bacteria ( $S$. mutans and F. nucleatum). The method involved fabrication of Na-zeoliteloaded (silane-modified Na-FAU zeolite, $2 \mathrm{wt} \%$ ) DA test pieces followed by treatment with a $0.01 \mathrm{M} \mathrm{AgNO}_{3}$ solution to exchange the $\mathrm{Na}$ zeolite counter-ions with silver. The Ag-charging did not affect visually the aesthetic appearance of the denture base materials, whereas the mechanical properties of the zeolite-loaded resins were within the standard requirements for such materials. The antimicrobial activity was associated with the sustained release of $\mathrm{Ag}$ from the materials. Antimicrobial tests of leached samples against $C$. albicans showed that the materials retained their activity for at least $45 \mathrm{~d}$, however the activity was lost after $60 \mathrm{~d}$ of incubation in distilled water. Cytotoxicity assay indicated that the introduction of $\mathrm{Ag}$ into DA did not result into additional cytotoxicity compared to neat DA. Considering the long-term antimicrobial activity of the Ag-charged dental acrylic resins containing zeolite, the potential for and the ease of silver recharging, the mechanical and color retention properties of the acrylic resins as well as the importance of silver as antimicrobial agent, the materials developed would be of great interest for prevention of denture-related oral diseases such as denture stomatitis. 


\section{Acknowledgements}

The authors would like to thank Erasmus + for providing funding for one of the authors (SR) to work at MMU. We would also like to thank Sama Belkhair, Sally Yip and Sarah Paintsil for providing some of the preliminary data, and Jodie Rowlands for assistance in the maintenance of the human cell line.

\section{Appendix A. Supplementary data}

Supplementary data associated with this article can be found, in the online version, at References

[1] P. E. Petersen, H. Ogawa, Periodontol. 200060 (2012) 15-39.

[2] D. W. Williams, T. Kuriyama, S. Silva, S. Malic, M. A. O. Lewis, Periodontol. 2000 55 (2011) 250-265.

[3] J. Boros-Majewska, N. Salewska, E. Borowski, S. Milewski, S. Malic, X.-Q. Wei, A. J. Hayes, M. J. Wilson, D. W. Williams, Med. Microbiol. Immun. 203 (2014) $341-355$

[4] J. Wen, C. K. Yeh, Y. Sun, J. Mater. Chem B 6 (2018) 1452-1457.

[5] D. Williams, M. Lewis, J. Oral Microbiol. 3 (2011) 5771-DOI: 10.3402/jom.v3i0.5771.

[6] L. F. D. Almeida, J. F. Paula, R. V. D. Almeida, D. W. Williams, J. Hebling, Y. W. Cavalcanti, Acta Odontol. Scand. 74 (2016) 393-398.

[7] L. Coulthwaite, J. Verran, Brit. J. Biomed. Sci. 64 (2007) 180-189.

[8] R. Nalcaci, I. Baran, Oral Surg. Oral Med. Oral Pathol. Oral Radiol. Endod. 105 (2008) e5-e9. 
[9] L. E. O'Donnell, D. Robertson, C. J. Nile, L. J. Cross, M. Riggio, A. Sherriff, D. Bradshaw, M. Lambert, J. Malcolm, M. J. Buijs, E. Zaura, V. Crielaard, B. W. Brandt, G. Ramage, Plos One 10 (2015) e0137717.

[10] B. Shi, T. Wu, J. McLean, A. Edlund, Y. Young, X. He, H. Lv, X. Zhou, W. Shi, H. Li, R. Lux, mSphere 1 (2016) e00215-16.

[11] R. Muenz, Aging and Demographic Change in European Societies: Main Trends and Alternative Policy Options. Societal protection, The World Bank, Washington, 2007.

[12] R. van Noort, Introduction to Dental Materials, $2^{\text {nd }}$ Edition, Mosby, London, 2002.

[13] R. P. Kusy, J.Q. Whitley, S. Kalachandra, Polymer 42 (2001) 2585-2595.

[14] J. M. Correa, M. Mori, H. L. Sanches, A. D. da Cruz, E. Poiate Jr., I. A. V. P. Poiate, Int. J. Biomater. (2015) DOI:10.1155/2015/485275.

[15] D. K. Morales, D. A. Hogan, Plos Pathog. 6 (2010) e1000886.

[16] A. Y. Peleg, D. A. Hogan, E. Mylonakis, Nat. Rev. Microbiol. 8 (2010) 340349.

[17] T. Pereira-Cenci, W. J. da Silva, M. S. Cenci, A. A. Cury, Int. J. Prosthodont. 23 (2010) 239-242.

[18] F. R. Teles, P. R. Teles, A. Sachdeo, N. G. Uzel, X. Q. Song, G. Torresyap, M. Singh, A. Papas, A. D. Haffajee, S. S. Socransky, J. Periodontol. 83 (2012) 11391148.

[19] H. Xu, T. Sobue, A. Thompson, Z. Xie, K. Poon, A. Ricker, J. Cervantes, P. I. Diaz and A. Dongari-Bagtzoglou, Cell Microbiol. 16 (2013) 214-231. 
[20] Y. W. Cavalcanti, D. J. Morse, W. J. da Silva, A. A. Del-Bel-Cury, X. Wei, M. Wilson, P. Milward, M. Lewis, D. Bradshaw, D. W. Williams, Biofouling 31 (2015) 27-38.

[21] R. S. Bedi, R. Cai, C. O'Neill, D. E. Beving, S. Foster, S. Guthrie, W. Chen, Y. Yan, Microporous Mesoporous Mater. 151 (2012) 352-357.

[22] S. Chernousova, M. Epple, Angew. Chem. Int. Ed. 52 (2013) 1636-1653.

[23] D. C. M. Kulatunga, S.H.S. Dananjaya, G. I. Godahewa, J. Lee, M. De Zoysa, Med. Mycol. 55 (2017) 213-222.

[24] V.P. Valtchev, K.N. Bozhilov, J. Phys. Chem. B 108 (2004) 15587-15598.

[25] S. Belkhair, M. Kinninmonth, L. Fisher, B. Gasharova, C.M. Liauw, J. Verran, B. Mihailova, L. Tosheva, RSC Adv. 5 (2015) 40932-40939.

[26] S. Malic, K. E. Hill, J. R. Ralphs, A. Hayes, D. W. Thomas, A. J. Potts, D. W. Williams, Oral Microbiol. Immun. 22 (2007) 188-194.

[27] L. Xu, A. Y. Hui, E. Albanis, M. J. Arthur, S. M. O'Byrne, W. S. Blaner, P. Mukherjee, S. L. Friedman, F. J. Eng, Gut. 54 (2005) 142-151.

[28] T. Mosmann, J. Immunol. Methods 65 (1983) 55-63.

[29] A. Yarborough, L. Cooper, I. Duqum, G. Mendonça, K. McGraw, L. Stoner, J. Prosthodon. 25 (2016) 288-301.

[30] M. H. Figueiral, P. Fonseca, M. M. Lopes, E. Pinto, T. Pereira-Leite, B. Sampaio-Maia, Open Dent. J. 9 (2015) 46-51.

[31] I. Sivakumar, K. S. Arunachalam, S. Sajjan, A. V. Ramaraju, B. Rao, B. Kamaraj, J. Prosthodon. 23 (2014) 284-290.

[32] J. Wen. F. Jiang, C. K. Yeh, Y. Sun, Colloids Surf. B 140 (2016) 19-27. 
[33] R. Jadhav, S. Bhide, B. L. V. Prasad and J. Shimpi, Eur. J. Prosthodon. 4 (2016) 45-50.

[34] L. S. Acosta-Torres, I. Mendieta, R. E. Nuñez-Anita, M. Cajero-Juárez, V. M. Castaño, Int. J. Nanomed. 7 (2012) 4777-4786.

[35] D. T. de Castro, M. L. C. Valente, C. H. L. da Silva, E. Watanabe, R. L. Siqueira, M. A. Schiavon, O. L. Alves, A. C. Dos Reis, Arch. Oral Biol. 67 (2016) $46-53$

[36] C. Fan, L. Chu, H. Ralph Rawls, B. K. Norling, H. L. Cardenas, K. Whang, Dent. Mater. 27 (2011) 322-328.

[37] J. H. Lee, A. El-Fiqia, J. K. Jo, D. A. Kim, S. C. Kim, S. K. Jun, H. W. Kim, H. H. Lee, Dent. Mater. 32 (2016) 1564-1574.

[38] W. Cao, Y. Zhang, X. Wang, Y. Chen, Q. Li, X. Xing, Y. Xiao, X. Peng, Z. Ye, J. Mater. Sci. Mater. Med. 28 (2017) 103.

[39] A. Malakhov, J. Wen, B. X. Zhang, H. Wang, H. Geng, X. D. Chen, Y. Sun, C. K. Yeh, Oral Dis. 22 (2016) 391-398.

[40] T. Dietrich, I. Webb, L. Stenhouse, A. Pattni, D. Ready, K. L. Wanyonyi, S. White, J. E. Gallagher, Brit. Dent. J. 222 (2017) 381-385.

[41] E. O. Oyetola, F. J. Owotade, G. A. Agbelusi, O. A. Fatusi, A. A. Sanusi, BMC Oral Health 15:24 (2015).

[42] D. Manger, M. Walshaw, R. Fitzgerald, J. Doughty, K. L. Wanyonyi, S. White, E. Gallagher, Brit. Dent. J. 222 (2017) 527-533. 


\section{Figure captions}

Figure 1. Digital images of DA resins: (a) from left to right: DA and Ag-FAU loaded DA at loadings of $0.2 \mathrm{wt} \%, 0.4 \mathrm{wt} \%, 0.7 \mathrm{wt} \%$ and $2.0 \mathrm{wt} \%$, and (b) from left to right: DA, DA/Ag-treated and DAZ/Ag-treated (2 wt $\%$ Na-FAU).

Figure 2. Antimicrobial activity of control (DA), DA/Ag-treated and DAZ/Ag-treated coupons against: (a) C. albicans NCYC 1363, (b) C. albicans 135 BM2/94, (c) S. mutans NCTC 10832 and (d) F. nucleatum KS 515-14.

Figure 3.

Absorbance readings from MTT assay performed on growth media used in the culture of LX-2 human hepatic cells in the presence of DA or DAZ/Ag-treated. There is no significant difference $(\mathrm{p}>0.05)$ in the absorbance, demonstrating no additional cytotoxicity of the DAZ/Ag-treated compared to DA. $n=3$ independent experiments. NS $=$ no significant difference.

Figure 4. Concentrations of Ag released from DAZ/Ag-treated coupons over 45 days.

Figure 5. Antimicrobial activity of DA coupons and DAZ/Ag-treated coupons against C. albicans $135 \mathrm{BM} 2 / 94$ after $45 \mathrm{~d}$ and after $60 \mathrm{~d}$ of incubation in distilled water; the coupons were transferred to fresh distilled water at $1,2,3,4,7,14,30$ and $45 \mathrm{~d}$ as described in experimental. 
Table 1. Flexural modulus and flexural strength values obtained (standard deviations are in parenthesis) for control DA and DA loaded with $2 \mathrm{wt} \%$ zeolites: Na-FAU (Zeolite DA), silane-treated Na-FAU (Zeolite-silane DA) and DA loaded with silane-treated AgFAU (Silver zeolite-silane DA).

\begin{tabular}{ccc}
\hline Specimen & $\begin{array}{c}\text { Flexural Modulus }(\mathrm{n}=6) \\
(\mathrm{GPa})\end{array}$ & $\begin{array}{c}\text { Flexural Strength }(\mathrm{n}=6) \\
(\mathrm{MPa})\end{array}$ \\
\hline Control DA & $2.52(0.10)$ & $91.5(1.3)$ \\
Zeolite DA & $2.45(0.05)$ & $84.0(6.0)$ \\
Zeolite-silane DA & $2.65(0.04)$ & $87.0(3.1)$ \\
Ag zeolite-silane DA & $2.82(0.12)$ & $83.3(6.4)$ \\
\hline
\end{tabular}



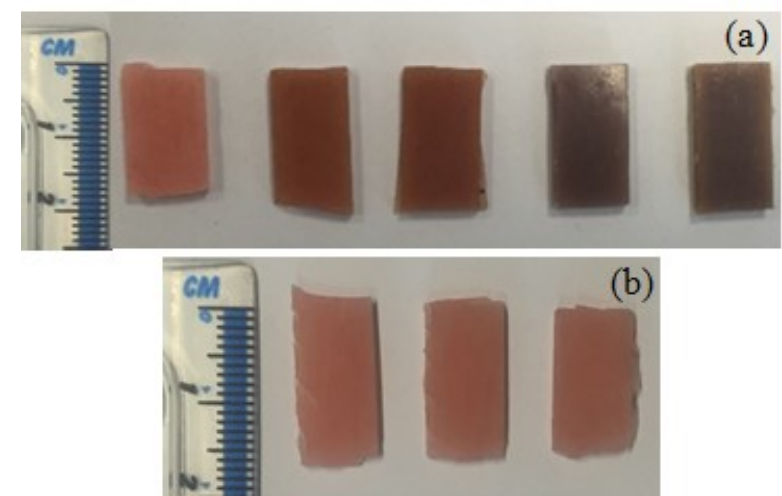

Figure 1 

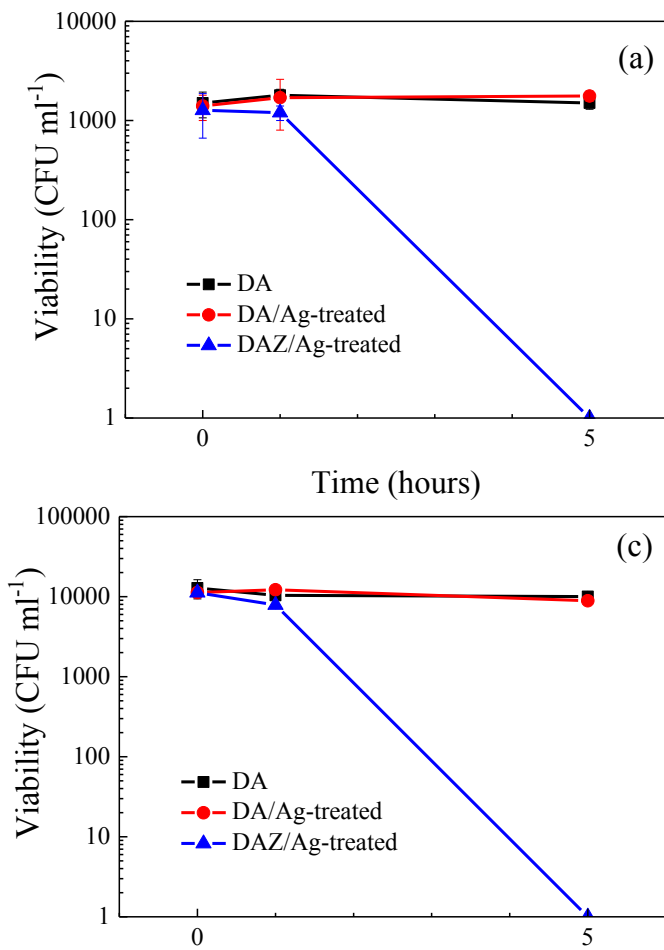

Time (hours)

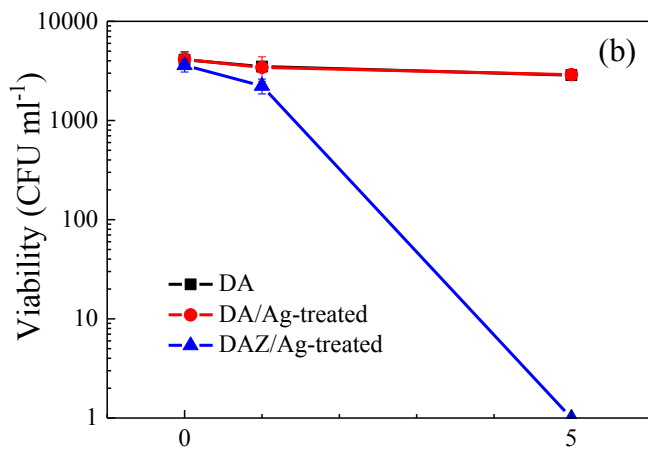

Time (hours)

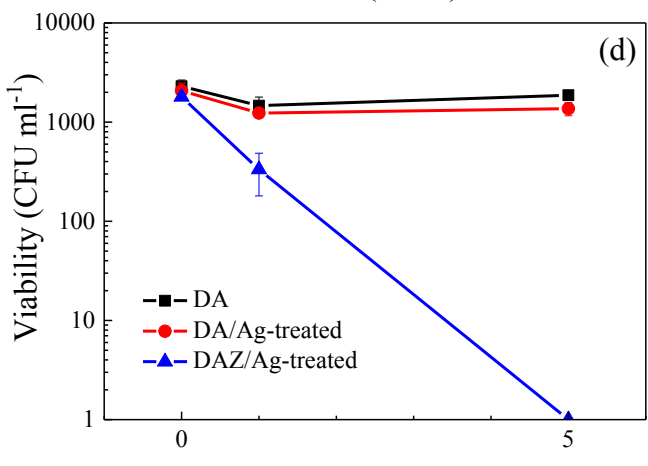

Time (hours)

Figure 2 


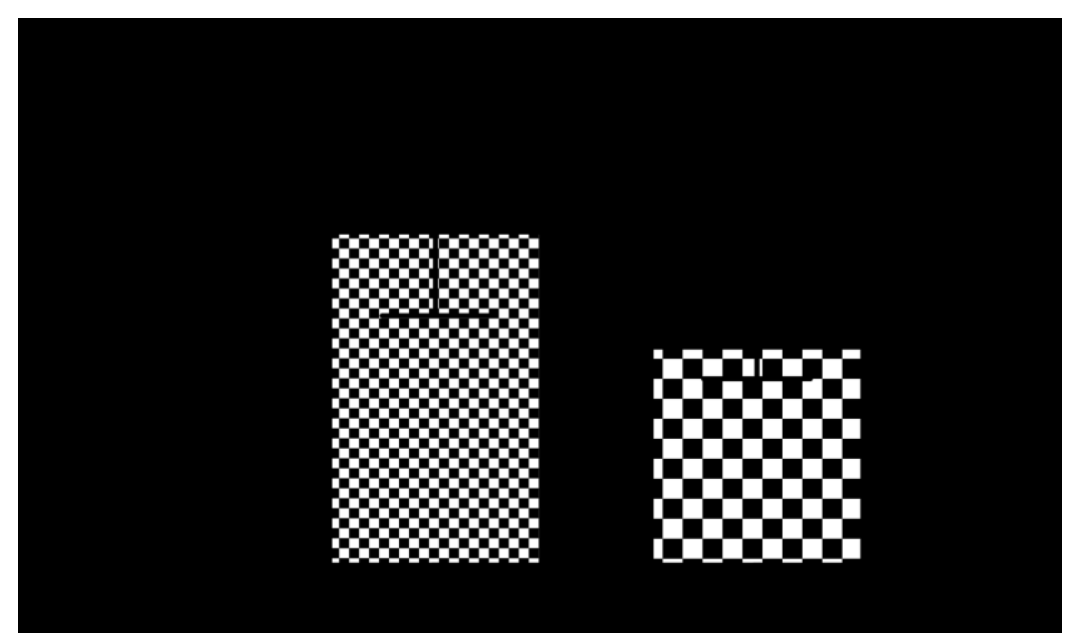

Figure 3 


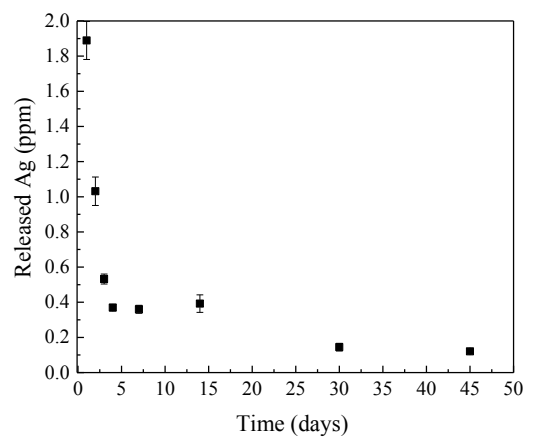

Figure 4 


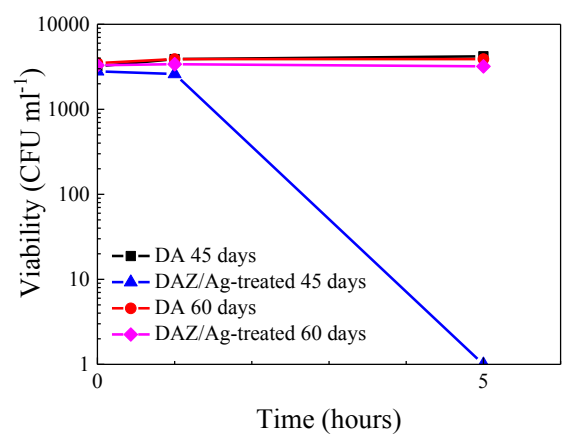

Figure 5 